\title{
Magnetic field-induced "mirage" gap in an Ising superconductor
}

\author{
Gaomin Tang, ${ }^{1}$ Christoph Bruder, ${ }^{1}$ and Wolfgang Belzig ${ }^{2}$ \\ ${ }^{1}$ Department of Physics, University of Basel, Klingelbergstrasse 82, CH-4056 Basel, Switzerland \\ ${ }^{2}$ Fachbereich Physik, Universität Konstanz, D-78457 Konstanz, Germany
}

\begin{abstract}
Superconductivity is commonly destroyed by a magnetic field due to orbital or Zeeman-induced pair breaking. Surprisingly, the spin-valley locking in a two-dimensional superconductor with spin-orbit interaction makes the superconducting state resilient to large magnetic fields. We investigate the spectral properties of such an Ising superconductor in a magnetic field taking into account disorder. The interplay of the in-plane magnetic field and the Ising spin-orbit coupling leads to noncollinear effective fields. We find that the emerging singlet and triplet pairing correlations manifest themselves in the occurrence of "mirage" gaps: at (high) energies of the order of the spin-orbit coupling strength, a gap-like structure in the spectrum emerges that mirrors the main superconducting gap. We show that these mirage gaps are signatures of the equal-spin triplet finite-energy pairing correlations and due to their odd parity are sensitive to intervalley scattering.
\end{abstract}

Introduction.- Superconductivity in two-dimensional materials is a rising topic [1] since these bear a great potential to host new pairing states due to their high chemical flexibility and the possibility to combine different materials in van der Waals heterostructures [2]. Monolayer transition-metal dichalcogenides were recently shown to be two-dimensional materials [3-6] with strong spin-orbit effects. Due to the broken in-plane inversion symmetry, the spin-orbit coupling arising from the heavy transition-metal atoms gives rise to a valley-dependent Zeeman-like spin splitting [6, 7]. Nevertheless, time-reversal symmetry is preserved because the internal field is opposite in the $K$ and $K^{\prime}$ valleys. Since this Zeemanlike field points out-of-plane, it was termed Ising spin-orbit coupling (ISOC) [8-10]. In such materials superconductivity has been shown to occur and is believed to be of $s$-wave type with possible admixtures of triplet pairing channels.

This so-called Ising superconductivity was experimentally realized from the few-layer down to the monolayer regime in various transition-metal dichalcogenides [9--25]. Since the electrons are confined to a two-dimensional plane, the orbital pair-breaking effect from an in-plane magnetic field is eliminated [26]. The presence of the ISOC lifts the spin degeneracy in the two valleys and this results in a considerably enhanced in-plane critical magnetic field [27-29] beyond the Pauli limit [30, 31]. Theoretical studies have mainly focused on the phase diagram [32-38], the occurrence of parity-mixed superconductivity [33, 35, 39, 40], topological superconductivity [8, 41,43], or transport problems [8, 44, 45]. In particular, the influence of scattering on the $s$-wave gap was investigated [32, 35, 36]. Moreover, due to the ISOC an inplane magnetic field can mediate the conversion from singlet Cooper pairs to equal-spin triplet pairs [33, 35, 37, 39].

In this Letter, we discuss the emergence of finite-energy pairing correlations in an Ising superconductor subject to an in-plane magnetic field. We show that these correlations are accompanied by the appearance of two symmetric mirages of the main superconducting gap shifted to a finite energy [See Figs. 1. (b) and 1.c)]. This picture is confirmed by relating the mirage gaps to finite-energy pairing that results from a subtle interplay between noncollinear spins and the valley degree of freedom. Using a fully self-consistent approach, we show that the intervalley scattering due to nonmagnetic impurities destroys the mirage gaps.

Hamiltonian.- For an Ising superconductor with a spinsinglet $s$-wave pairing gap $\Delta$, the effective Bogoliubov-de Gennes Hamiltonian near one of the valleys can be written in the Nambu basis $\left(c_{\boldsymbol{k}, \uparrow}, c_{\boldsymbol{k}, \downarrow}, c_{-\boldsymbol{k}, \uparrow}^{\dagger}, c_{-\boldsymbol{k}, \downarrow}^{\dagger}\right)$ as

$$
H_{\mathrm{BdG}}=\left[\begin{array}{cc}
H_{0}(\boldsymbol{k}) & \Delta i \sigma_{y} \\
-\Delta i \sigma_{y} & -H_{0}^{*}(-\boldsymbol{k})
\end{array}\right] .
$$

Here, $H_{0}$ is

$$
H_{0}(\boldsymbol{k}=\boldsymbol{p}+s \boldsymbol{K})=\xi_{\boldsymbol{p}} \sigma_{0}+s \beta_{\mathrm{so}} \sigma_{z}-B_{x} \sigma_{x},
$$

where $s \boldsymbol{K}$ is the position of the valley $K(s=+)$ or $K^{\prime}$ $(s=-)$ in momentum space and $\boldsymbol{p}$ is the deviation from the $\boldsymbol{K}\left(\boldsymbol{K}^{\prime}\right)$-point. Furthermore, $\xi_{\boldsymbol{p}}=p^{2} /(2 m)-\mu$ is the dispersion measured form the chemical potential $\mu$. The Pauli matrices $\sigma_{x}, \sigma_{y}$, and $\sigma_{z}$ act on the spin space and $\sigma_{0}$ is the unit matrix. The ISOC $\beta_{\text {so }}$ pins the electron spins to the out-ofplane. The in-plane magnetic field $B_{x}$ is along the $x$-direction and induces the Zeeman term $-B_{x} \sigma_{x}$. The prefactor $g_{L} \mu_{B} / 2$ with the Landé g-factor $g_{L}$ and the Bohr magneton $\mu_{B}$ has been absorbed in $B_{x}$. Since the magnetic field $B_{x}$, which is valley symmetric, tends to tilt the electron spins in the $x$ direction, the spins are reoriented [See Fig. [1(a)]. The band splitting in the normal state is $2 \sqrt{\beta_{\mathrm{so}}^{2}+B_{x}^{2}}$.

Finite-energy pairing.- The general pairing-correlation function can be expressed as [28, 29]

$$
F(\boldsymbol{k}, \varepsilon)=\Delta\left[F_{0}(\boldsymbol{k}, \varepsilon) \sigma_{0}+\boldsymbol{F}(\boldsymbol{k}, \varepsilon) \cdot \boldsymbol{\sigma}\right] i \sigma_{y},
$$

where $F_{0}$ and $\boldsymbol{F}$, respectively, parametrize the singlet and triplet pairing correlations [46]. Using Eq. [3], the pairing wave function can be written as

$$
\begin{array}{r}
|\Psi\rangle=F_{0}(|\uparrow \downarrow\rangle-|\downarrow \uparrow\rangle)+F_{x}(|\downarrow \downarrow\rangle-|\uparrow \uparrow\rangle) \\
+i F_{y}(|\downarrow \downarrow\rangle+|\uparrow \uparrow\rangle)+F_{z}(|\uparrow \downarrow\rangle+|\downarrow \uparrow\rangle) .
\end{array}
$$

Here, the momentum dependence is omitted, for example, $|\boldsymbol{k} \uparrow,-\boldsymbol{k} \downarrow\rangle$ is abbreviated as $|\uparrow \downarrow\rangle$.

We first discuss the low-energy pairing that occurs around the Fermi energy. In the absence of a magnetic field $B_{x}$, the 

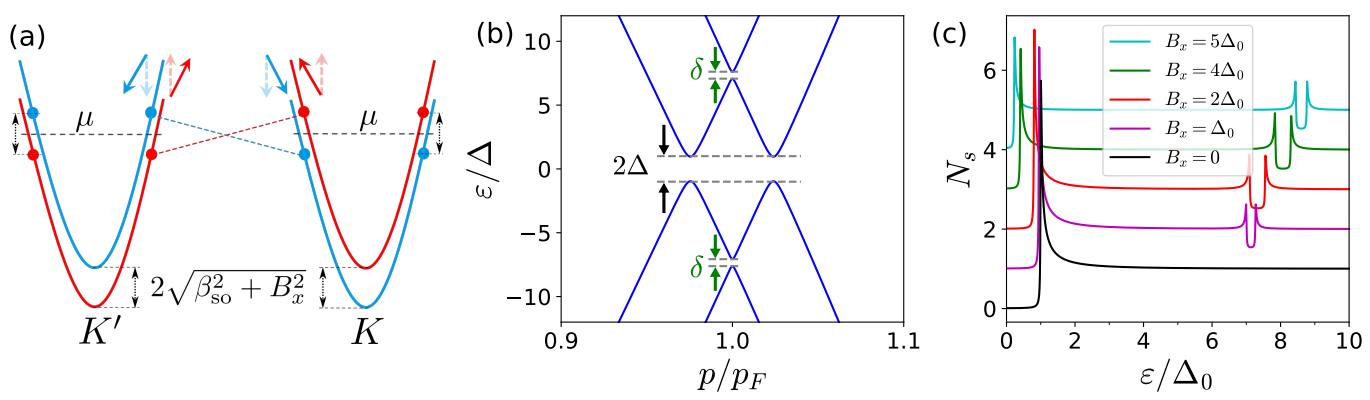

FIG. 1. (a) Schematic band structure in the normal state. The electrons near the $K$ and $K^{\prime}$ valleys are subject to the ISOC $\beta_{\text {so }}$, which pins the electron spins to the out-of-plane direction (dashed arrows), and an in-plane magnetic field $B_{x}$. For finite $B_{x}$, the spin directions are reoriented (solid arrows) (b) Quasiparticle energy spectrum of Eq. (1) near the Fermi momentum $p_{F}$ with $\beta_{\text {so }}=7 \Delta, B_{x}=2 \Delta$ and $\mu=150 \Delta$. The mirage gaps $\delta$ are shifted images of the main superconducting gap. (c) Density of states $N_{s}$ for different $B_{x}$ in the clean limit. All lines for finite $B_{x}$ have been offset for better visibility. Here, $\beta_{\text {so }}=7 \Delta_{0}$ and $T=0.1 T_{c 0}$, where $\Delta_{0}$ and $T_{c 0}$ are, respectively, the zero-temperature gap and transition temperature in the absence of a magnetic field.

ISOC field results in opposite energy splittings in the two valleys so that the amplitude of the pairing state $|\uparrow \downarrow\rangle$ is different from that of $|\downarrow \uparrow\rangle$ except at the Fermi momentum. Hence, in addition to the standard singlet pair amplitude $\propto F_{0}$, a pairing state $|\uparrow \downarrow\rangle+|\downarrow \uparrow\rangle$ is created, i.e., $F_{z}$ is finite [8, 28, 29, 35]. This pair amplitude $F_{z}$, which is due to the ISOC, has the form $F_{z} \propto s \beta_{\text {so }} \xi_{p}[8,39$, 46] and is odd in the valley index. In the presence of $B_{x}$, the electron spins are reoriented so that equalspin pairing states $|\uparrow \uparrow\rangle_{x}$ and $|\downarrow \downarrow\rangle_{x}$ emerge around the Fermi energy. Here, the subscript ' $x$ ' denotes the spin states in the $x$-direction. This leads to the triplet states $|\uparrow \uparrow\rangle_{x}+|\downarrow \downarrow\rangle_{x}$ and $|\uparrow \uparrow\rangle_{x}-|\downarrow \downarrow\rangle_{x}$, which in the $z$-basis take the form $|\uparrow \uparrow\rangle+|\downarrow \downarrow\rangle$ and $|\uparrow \downarrow\rangle+|\downarrow \uparrow\rangle$, respectively [46].

The interplay between ISOC and an in-plane magnetic field leads to a new feature, viz., finite-energy pairing correlations. A qualitative illustration is provided in Fig. 1 1 a) that shows the schematic band structure with electrons at Fermi momenta $p_{F}= \pm \sqrt{2 m \mu}$ as blue and red dots. Near the Fermi momentum, the electron at $|\boldsymbol{k} \uparrow\rangle_{x}\left(|\boldsymbol{k} \downarrow\rangle_{x}\right)$ can pair with the electron at $|-\boldsymbol{k} \downarrow\rangle_{x}\left(|-\boldsymbol{k} \uparrow\rangle_{x}\right)$ as indicated by the dashed lines in Fig. 11.a). As a consequence, there is a coexistence of the singlet state $|\uparrow \downarrow\rangle_{x}-|\downarrow \uparrow \uparrow\rangle_{x}$ and the triplet state $|\uparrow \downarrow\rangle_{x}+|\downarrow \uparrow\rangle_{x}$, which in the $z$-basis is $|\downarrow \downarrow\rangle-|\uparrow \uparrow\rangle$ [46]. Similarly, there are also equalspin triplet states $|\downarrow \downarrow\rangle$ and $|\uparrow \uparrow\rangle$ near the Fermi momenta in the $z$-direction. This can give rise to the equal-spin triplet states $|\downarrow \downarrow\rangle-|\uparrow \uparrow\rangle$ and $|\downarrow \downarrow\rangle+|\uparrow \uparrow\rangle$. We term these finite-energy pairing states, since the two electrons forming a Cooper pair have opposite energies with respect to the Fermi energy and are separated in energy by about $2 \sqrt{\beta_{\mathrm{so}}^{2}+B_{x}^{2}}$, which is typically much larger than $2 \Delta$.

The pairing states and the symmetries of the corresponding pair amplitudes are summarized in Table $]$ The pair amplitude $F_{x}$ is odd in time, since $B_{x}$ breaks the time-reversal symmetry. The overall antisymmetry of the Cooper pair wave function is ensured by the parity under exchanging the arguments of spin, valley, and time [47-50]. The symmetries of the amplitudes $F_{x}$ and $i F_{y}$ that are even and odd, respectively, under exchanging the valley indices, will become clear later in

\begin{tabular}{|c||c|c|c|c|c|}
\hline & $\begin{array}{c}\text { pairing states } \\
\text { (zero energy) }\end{array}$ & $\begin{array}{c}\text { pairing states } \\
\text { (finite energy) }\end{array}$ & spin & valley & time \\
\hline$F_{0}$ & $|\uparrow \downarrow\rangle-|\downarrow \uparrow\rangle$ & $|\uparrow \downarrow\rangle-|\downarrow \uparrow\rangle$ & singlet & even & even \\
\hline$F_{x}$ & $\times$ & $|\downarrow \downarrow\rangle-|\uparrow \uparrow\rangle$ & triplet & even & odd \\
\hline$i F_{y}$ & $|\downarrow \downarrow\rangle+|\uparrow \uparrow\rangle$ & $|\downarrow \downarrow\rangle+|\uparrow \uparrow\rangle$ & triplet & odd & even \\
\hline$F_{z}$ & $|\uparrow \downarrow\rangle+|\downarrow \uparrow\rangle$ & $\times$ & triplet & odd & even \\
\hline
\end{tabular}

TABLE I. Pairing states at zero and finite energy of an Ising superconductor subject to an in-plane magnetic field. Zero-energy $F_{x}$ pairing and finite-energy $F_{z}$ pairing do not exist. The symmetries of the pair amplitudes are characterized by the parity under exchanging the arguments of spin, valley, and time.

Eq. (9) from the quasiclassical Green's function formalism.

The presence of the finite-energy pairing correlations is reflected in the density of states (DOS). The quasiparticle energy spectrum for $\Delta \ll \beta_{\text {so }} \ll \mu$ is shown in Fig. 11 b). In addition to the main superconducting gap, there are mirage gaps of size $\delta$ appearing at the Fermi momentum. These mirage gaps can be interpreted as an image of the main superconducting gap shifted by the effective field and is a hallmark of the finite-energy pairing correlations. Note that the DOS is finite in the mirage gaps, since in each gap only one band in each valley participates in the finite-energy pairing [See Fig. 11(a)]. The mirage gaps are located at $\pm \varepsilon_{0}$ with $\varepsilon_{0}=\left(\varepsilon_{1}+\varepsilon_{2}\right) / 2$, where $\varepsilon_{1(2)}=\sqrt{\beta_{\mathrm{so}}^{2}+\left(B_{x} \pm \Delta\right)^{2}}$ are the eigenvalues of the Hamiltonian $H_{\mathrm{BdG}}$ at $\xi_{\boldsymbol{p}}=0$. Their widths are $\delta=\varepsilon_{1}-\varepsilon_{2}$. The location and width of mirage gap can be used to experimentally extract the strength of the ISOC. This is necessary to estimate the upper critical magnetic fields at low temperatures, which are too large to be measured directly at present. For the case without ISOC $\left(\beta_{\mathrm{so}}=0\right)$, we arrive at $\varepsilon_{0}= \pm \Delta$ and $\delta=2 B_{x}$ and this reduces to the well-known Zeeman splitting between the spin-up and spin-down electrons. This splitting suppresses the formation of Cooper pairs and results in the paramagnetic limit of superconductivity [30, 31]. For $\beta_{\text {so }} \gg \Delta$, the mirage gaps are clearly separated from the main 
gaps; they appear around the energy

$$
\varepsilon_{0} \approx \sqrt{\beta_{\mathrm{so}}^{2}+B_{x}^{2}},
$$

and have widths

$$
\delta \approx 2 \Delta B_{x} / \sqrt{\beta_{\mathrm{so}}^{2}+B_{x}^{2}} .
$$

It can be inferred that in the absence of $B_{x}, \delta$ vanishes, i.e., there is no finite-energy pairing. Note that for $B_{x} \gtrsim \beta_{\text {so }}$ pair breaking sets in so that the main superconducting gap vanishes and consequently so do the mirage gaps.

Figure 1 (c) shows the superconducting DOS $N_{s}$ (normalized to that of the normal state) for different in-plane magnetic fields in the clean limit. The curves are calculated using the quasiclassical Green's function formalism. The DOS is 0.5 inside each mirage gap, since only one band in each valley participates in the pairing. On increasing the magnetic field, the superconducting gap $\Delta$ decreases, while the mirage gap $\delta$ shows a nonmonotonic behavior and first increases and then decreases. This is due to the interplay between the gap $\Delta$ and the magnetic field $B_{x}$, as indicated by Eq. (6). Below a certain value of $B_{x}$, the decreasing slope of $\Delta$ is smaller than the increasing slope of $B_{x}$ [See Figs. S4(b) and S4(d) in the Supplemental Material [46]], so that the increase of $B_{x}$ dominates. However, above this value of $B_{x}$, the decrease of $\Delta$ dominates and $\delta$ decreases.

Quasiclassical Green's function.- We now describe the formalism used to calculate the DOS and the pair amplitudes. Since the quasiclassical formalism concentrates on the phenomena close to the Fermi surface [51-54], it can be applied to the situation where both the superconducting gap and the ISOC are much smaller than the Fermi energy. The general structure of the quasiclassical Green's function in Nambu space is [54, 55]

$$
\hat{g}(\hat{\boldsymbol{k}}, \varepsilon)=\left[\begin{array}{cc}
g_{0} \sigma_{0}+\boldsymbol{g} \cdot \boldsymbol{\sigma} & \left(f_{0} \sigma_{0}+\boldsymbol{f} \cdot \boldsymbol{\sigma}\right) i \sigma_{y} \\
\left(\bar{f}_{0} \sigma_{0}+\overline{\boldsymbol{f}} \cdot \boldsymbol{\sigma}^{*}\right) i \sigma_{y} & \bar{g}_{0} \sigma_{0}+\overline{\boldsymbol{g}} \cdot \boldsymbol{\sigma}^{*}
\end{array}\right],
$$

where $\hat{k}$ denotes the direction of momentum $\boldsymbol{k}$ and $\varepsilon$ is the quasiparticle energy with respect to the Fermi energy. The bar operation is defined as $\bar{q}(\hat{\boldsymbol{k}}, \varepsilon)=q\left(-\hat{\boldsymbol{k}},-\varepsilon^{*}\right)^{*}$ with $q \in$ $\left\{g_{0}, f_{0}, \boldsymbol{g}, \boldsymbol{f}\right\}$. The anomalous Green's functions $f_{0}$ and $\boldsymbol{f}$, respectively, correspond to $F_{0}$ and $\boldsymbol{F}$ in Eq. (3). The DOS $N_{s}$ is given by $\operatorname{Re}\left(g_{0}\right)$.

The Eilenberger equation for a homogeneous system reads [51, 54]

$$
\left[\varepsilon \sigma_{0} \tau_{3}-\hat{\Delta}-\hat{\nu}-\hat{\Sigma}(\varepsilon), \hat{g}\right]=0,
$$

with the order parameter term $\hat{\Delta}=\Delta i \sigma_{y} \tau_{2}$. The Pauli matrices $\tau_{1} \tau_{2}$, and $\tau_{3}$ act on the Nambu space and $\tau_{0}$ is the corresponding unit matrix. The ISOC and Zeeman terms are included in $\hat{\nu}$ with $\hat{\nu}=s \beta_{\text {so }} \sigma_{z} \tau_{3}-B_{x} \sigma_{x} \tau_{0}$. Nonmagnetic impurities are taken into account using the self-consistent Born approximation with $\hat{\Sigma}(\varepsilon)=-i \Gamma\langle\hat{g}(\hat{\boldsymbol{k}}, \varepsilon)\rangle$, where $\Gamma$ is the intervalley impurity scattering rate and $\langle\cdots\rangle$ denotes the average over the Fermi momentum direction. It has been theoretically demonstrated that nonmagnetic intervalley scattering can suppress the upturn of the in-plane critical magnetic field in the low temperature region [32, 35]. According to Anderson's theorem [56], intravalley nonmagnetic scattering has no effect for an $s$-wave superconductor, which is the case here. By combining with $\operatorname{Tr}(\hat{g})=0$ and the normalization condition $\hat{g} \hat{g}=\sigma_{0} \tau_{0}$, all the components of $\hat{g}$ can be obtained. In particular, $f_{x}$ and $f_{y}$ can be, respectively, written as

$$
f_{x}=a \tilde{\varepsilon} B_{x}, \quad f_{y}=a \text { is } \beta_{\mathrm{so}} B_{x},
$$

where $\tilde{\varepsilon}=\varepsilon+i \Gamma g_{0}$ and $a$ is fixed by the normalization condition. The derivation and calculation details can be found in the Supplemental Material [46]. Equation (9) shows that the $i f_{y}$ pairing is a consequence of the interplay between ISOC and the in-plane magnetic field. We can also deduce from Eq. (9) that $f_{x}$ and $f_{y}$ are even and odd with respect to the valley index $s$, respectively. This confirms the valley symmetries shown in Table The amplitudes of $f_{x}$ and $i f_{y}$ are not equal around $\varepsilon= \pm \varepsilon_{0}$ and the difference lies in the finite-energy pairing $|\uparrow \downarrow\rangle_{x}+|\downarrow \uparrow\rangle_{x}$. Since only an in-plane magnetic field is applied and the dispersion $\xi_{p}$ is integrated over in the quasiclassical formalism [33, 54], the quasiclassical pair amplitude $f_{z}$ vanishes.

Numerical results and discussion.- To unveil the microscopic mechanism, we present self-consistent numerical results at different intervalley scattering strengths $\Gamma$ in Fig. 2. To account for inelastic processes, a Dynes broadening parameter $\eta=0.01 \Delta_{0}$ has been added, $\varepsilon \rightarrow \varepsilon+i \eta$ [57]. Figure 2]b) shows that $\operatorname{Im}\left(f_{0}\right)$ is finite near $\varepsilon= \pm \varepsilon_{0}$; this is the consequence of finite-energy singlet pairing $|\uparrow \downarrow\rangle_{x}-|\downarrow \uparrow\rangle_{x}$. Finiteenergy equal-spin pairing correlations $|\downarrow \downarrow\rangle \mp|\uparrow \uparrow\rangle$ are visible in Figs.2 2(c) and 2(d). An electron in $K$ valley in state $|\boldsymbol{k} \uparrow\rangle$ near energy $\varepsilon=\varepsilon_{0}$ pairs with the electron in state $|-\boldsymbol{k} \uparrow\rangle$ with energy $-\varepsilon$ forming the pairing state $|\uparrow \uparrow\rangle$ [See Fig. 11a)]. Consequently, $\operatorname{Im}\left(f_{x}\right)$ is negative while $\operatorname{Im}\left(i f_{y}\right)$ is positive around $\varepsilon=\varepsilon_{0}$. Similarly, $\operatorname{Im}\left(f_{x}\right)$ and $\operatorname{Im}\left(i f_{y}\right)$ are positive around $\varepsilon=-\varepsilon_{0}$ due to the pairing $|\downarrow \downarrow\rangle$. The pair amplitude $\operatorname{Im}\left(f_{x}\right)$ is odd in energy due to the time-reversal symmetry breaking induced by the in-plane magnetic field. The finite values of $\operatorname{Im}\left(i f_{y}\right)$ around the Fermi energy $(\varepsilon=0)$ are a manifestation of the zero-energy pairing $|\uparrow \uparrow\rangle_{x}+|\downarrow \downarrow\rangle_{x}$. The finite values of $f_{x}$ near $\varepsilon= \pm \Delta$ are due to the Dynes broadening used in the numerical calculation.

We now turn to the discussion of nonmagnetic intervalley scattering effects. As can be seen from the DOS [Fig. 2(a)] and the singlet pair amplitude [Fig. 2(b)], the superconducting gap decreases with increasing impurity scattering strength [32, 35]. Meanwhile, the finite-energy pairing correlations get suppressed as well and are more sensitive to the impurity scattering than the zero-energy singlet pairing. Because of the suppression of the finite-energy pairing correlations, the DOS inside the mirage gaps increases. It can be seen from Figs. 2(c) and 2(d) that the finite-energy pairing correlations almost vanish for $\Gamma=2 \Delta_{0}$. The effect of nonmagnetic intervalley scattering can be explained as follows. First, the mirage gap is proportional to the main superconducting gap which is suppressed due to the intervalley scattering. Since 

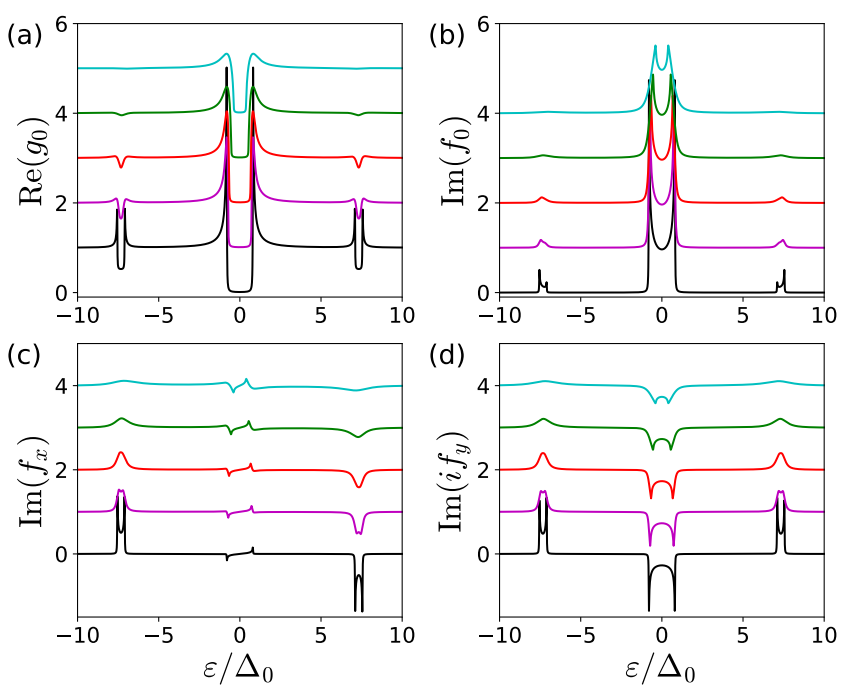

FIG. 2. Effects of intervalley scattering. Various components of the Green's function $\hat{g}$ at different intervalley scattering strengths (from bottom to top: $\Gamma=0,0.2 \Delta_{0}, 0.4 \Delta_{0}, \Delta_{0}, 2 \Delta_{0}$ ) in valley $K$ with $\beta_{\text {so }}=7 \Delta_{0}, B_{x}=2 \Delta_{0}$ and $T=0.1 T_{c 0}$. All curves for finite $\Gamma$ have been offset for better visibility. For valley $K^{\prime}$, the sign of $f_{y}$ reverses, while the signs of $g_{0}, f_{0}$ and $f_{x}$ remain unchanged.

nonmagnetic scattering is spin-conserving, it connects electron states from different valleys with the same out-of-plane spin direction. Due to the spin reorientation by the in-plane magnetic field, there is scattering between $|\boldsymbol{k} \uparrow\rangle_{x}$ and $|-\boldsymbol{k} \downarrow\rangle_{x}$ which in turn reduces the effect of the magnetic field. The effective magnetic field becomes $\tilde{B}_{x}=B_{x}+i \Gamma g_{+, x}$ with $g_{+, x}$ characterizing the in-plane magnetization induced by $B_{x}[46]$. This further reduces the mirage gap width. Moreover, because of the finite DOS in one valley inside the mirage gap of the other valley, nonmagnetic impurity scattering leads to an imaginary part of the energy $\sim \Gamma g_{0} \gtrsim \Gamma / 2$, so that the coherence peaks of the mirage gaps are smeared. A more detailed analytical treatment of the impurity effect is presented in the Supplemental Material [46].

Here, we only consider an $s$-wave order parameter in the singlet channel. The existence of singlet-triplet mixing of the order parameter has been discussed [20, 22, 35, 40]. The presence of a triplet component and singlet-triplet mixing could possibly lead to new and interesting properties of the mirage gaps.

The mirage gaps appear to be similar to the hybridization gaps in a two-band superconductor [58]. However, the underlying physics is quite different. The hybridization gaps are due to single-quasiparticle scattering between two superconducting bands, while the mirage gaps are the consequence of finite-energy pairing. Moreover, the mirage gaps are associated with triplet pairing correlations, while the correlations in Ref. [58] are of singlet type. Both the mirage gaps and hybridization gaps relate to the appearance of odd-frequency pairing.

It is interesting to compare the finite-energy pairing state with the Fulde-Ferrell-Larkin-Ovchinnikov (FFLO) state [59, 60] in superconductors with large magnetic fields. For the FFLO state, due to the Zeeman splitting, two electrons at the Fermi surface with the same energy can only pair with each other at the cost of a finite center-of-mass momentum. In contrast to that, for the finite-energy pairing, the two electrons forming a Cooper pair have the opposite momentum at the cost of different energies.

Our findings should be experimentally accessible using tunneling spectroscopy [13, 14, 26, 61] in an Ising superconductor with moderate ISOC, such as $\mathrm{MoS}_{2}$ [1, 9, 11], by applying an in-plane magnetic field. For Ising superconductors with large ISOC, such as $\mathrm{NbSe}_{2}$ [10, 16, 20, 23], $\mathrm{WS}_{2}$ [15] and $\mathrm{TaS}_{2}$ [16], identifying the mirage gaps requires a relatively large magnetic field according to Eq. (6). This could be possibly realized using the magnetic exchange field from a ferromagnetic substrate [22, 23].

To conclude, we have identified the emergence of finiteenergy pairing correlations in an Ising superconductor subject to an in-plane magnetic field. The accompanying mirage gaps offer an experimental signature. The mirage gaps can also lead to equal-spin Andreev reflection at interfaces between an Ising superconductor and a normal or ferromagnetic metal. A Josephson junction between two Ising superconductors with noncollinear in-plane magnetic fields may host spin-polarized Andreev bound states inside the mirage gaps that can be detected by spin-resolved spectroscopy. The concept of the mirage gap thus offers a new perspective on the interplay between superconductivity and magnetism.

Acknowledgments.- G.T. and C.B. acknowledge financial support from the Swiss National Science Foundation (SNSF) and the NCCR Quantum Science and Technology. W.B. acknowledges funding by the Deutsche Forschungsgemeinschaft (DFG, German Research Foundation) - Project-ID 443404566 - SPP 2244.

[1] Y. Saito, T. Nojima, and Y. Iwasa, Highly crystalline 2D superconductors, Nature Reviews Materials 2, 16094 (2016)

[2] A. K. Geim and I. V. Grigorieva, Van der Waals heterostructures, Nature 499, 419 (2013)

[3] K. F. Mak, C. Lee, J. Hone, J. Shan, and T. F. Heinz, Atomically thin $\mathrm{MoS}_{2}$ : A new direct-gap semiconductor, Phys. Rev. Lett. 105, 136805 (2010)

[4] B. Radisavljevic, A. Radenovic, J. Brivio, V. Giacometti, and A. Kis, Single-layer $\mathrm{MoS}_{2}$ transistors, Nature Nanotechnology 6, 147 (2011).

[5] Q. H. Wang, K. Kalantar-Zadeh, A. Kis, J. N. Coleman, and M. S. Strano, Electronics and optoelectronics of twodimensional transition metal dichalcogenides, Nature Nanotechnology 7, 699 (2012)

[6] Z. Y. Zhu, Y. C. Cheng, and U. Schwingenschlögl, Giant spin-orbit-induced spin splitting in two-dimensional transitionmetal dichalcogenide semiconductors, Phys. Rev. B 84, 153402 (2011)

[7] D. Xiao, G.-B. Liu, W. Feng, X. Xu, and W. Yao, Coupled spin 
and valley physics in monolayers of $\mathrm{MoS}_{2}$ and other group-VI dichalcogenides, Phys. Rev. Lett. 108, 196802 (2012)

[8] B. T. Zhou, N. F. Q. Yuan, H.-L. Jiang, and K. T. Law, Ising superconductivity and Majorana fermions in transition-metal dichalcogenides, Phys. Rev. B 93, 180501(R) (2016)

[9] J. M. Lu, O. Zheliuk, I. Leermakers, N. F. Q. Yuan, U. Zeitler, K. T. Law, and J. T. Ye, Evidence for two-dimensional Ising superconductivity in gated $\mathrm{MoS}_{2}$, Science 350, 1353 (2015)

[10] X. Xi, Z. Wang, W. Zhao, J.-H. Park, K. T. Law, H. Berger, L. Forró, J. Shan, and K. F. Mak, Ising pairing in superconducting $\mathrm{NbSe}_{2}$ atomic layers, Nature Physics 12, 139 (2016)

[11] Y. Saito, Y. Nakamura, M. S. Bahramy, Y. Kohama, J. Ye, Y. Kasahara, Y. Nakagawa, M. Onga, M. Tokunaga, T. Nojima, Y. Yanase, and Y. Iwasa, Superconductivity protected by spin-valley locking in ion-gated $\mathrm{MoS}_{2}$, Nature Physics 12, 144 (2016)

[12] Y. Xing, K. Zhao, P. Shan, F. Zheng, Y. Zhang, H. Fu, Y. Liu, M. Tian, C. Xi, H. Liu, J. Feng, X. Lin, S. Ji, X. Chen, Q.-K. Xue, and J. Wang, Ising superconductivity and quantum phase transition in macro-size monolayer $\mathrm{NbSe}_{2}$, Nano Letters 17, $6802(2017)$.

[13] T. Dvir, F. Massee, L. Attias, M. Khodas, M. Aprili, C. H. L. Quay, and H. Steinberg, Spectroscopy of bulk and few-layer superconducting $\mathrm{NbSe}_{2}$ with van der Waals tunnel junctions, Nature Communications 9, 598 (2018)

[14] D. Costanzo, H. Zhang, B. A. Reddy, H. Berger, and A. F. Morpurgo, Tunnelling spectroscopy of gate-induced superconductivity in $\mathrm{MoS}_{2}$, Nature Nanotechnology 13, 483 (2018)

[15] J. Lu, O. Zheliuk, Q. Chen, I. Leermakers, N. E. Hussey, U. Zeitler, and J. Ye, Full superconducting dome of strong Ising protection in gated monolayer $\mathrm{WS}_{2}$, Proceedings of the National Academy of Sciences 115, 3551 (2018)

[16] S. C. de la Barrera, M. R. Sinko, D. P. Gopalan, N. Sivadas, K. L. Seyler, K. Watanabe, T. Taniguchi, A. W. Tsen, X. Xu, D. Xiao, and B. M. Hunt, Tuning Ising superconductivity with layer and spin-orbit coupling in two-dimensional transition-metal dichalcogenides, Nature Communications 9, $1427(2018)$

[17] E. Sohn, X. Xi, W.-Y. He, S. Jiang, Z. Wang, K. Kang, J.-H. Park, H. Berger, L. Forró, K. T. Law, J. Shan, and K. F. Mak, An unusual continuous paramagnetic-limited superconducting phase transition in 2D NbSe ${ }_{2}$, Nature Materials 17, 504 (2018)

[18] D. Rhodes, N. F. Yuan, Y. Jung, A. Antony, H. Wang, B. Kim, Y. che Chiu, T. Taniguchi, K. Watanabe, K. Barmak, L. Balicas, C. R. Dean, X. Qian, L. Fu, A. N. Pasupathy, and J. Hone, Enhanced superconductivity in monolayer $\mathrm{T}_{d}-\mathrm{MoTe}_{2}$ with tilted Ising spin texture (2019), arXiv:1905.06508

[19] J. Li, P. Song, J. Zhao, K. Vaklinova, X. Zhao, Z. Li, Z. Qiu, Z. Wang, L. Lin, M. Zhao, T. S. Herng, Y. Zuo, W. Jonhson, W. Yu, X. Hai, P. Lyu, H. Xu, H. Yang, C. Chen, S. J. Pennycook, J. Ding, J. Teng, A. H. Castro Neto, K. S. Novoselov, and J. Lu, Printable two-dimensional superconducting monolayers, Nature Materials 20, 181 (2021).

[20] C. woo Cho, J. Lyu, T. Han, C. Y. Ng, Y. Gao, G. Li, M. Huang, N. Wang, J. Schmalian, and R. Lortz, Distinct nodal and nematic superconducting phases in the 2D Ising superconductor $\mathrm{NbSe}_{2}$ (2020), arXiv:2003.12467

[21] M. Kuzmanović, T. Dvir, D. LeBoeuf, S. Ilić, D. Möckli, M. Haim, S. Kraemer, M. Khodas, M. Houzet, J. S. Meyer, M. Aprili, H. Steinberg, and C. H. L. Quay, Tunneling spectroscopy of few-monolayer $\mathrm{NbSe}_{2}$ in high magnetic field: Ising protection and triplet superconductivity (2021), arXiv:2104.00328.

[22] A. Hamill, B. Heischmidt, E. Sohn, D. Shaffer, K.-T. Tsai,
X. Zhang, X. Xi, A. Suslov, H. Berger, L. Forró, F. J. Burnell, J. Shan, K. F. Mak, R. M. Fernandes, K. Wang, and V. S. Pribiag, Two-fold symmetric superconductivity in few-layer $\mathrm{NbSe}_{2}$, Nature Physics 10.1038/s41567-021-01219-x (2021).

[23] K. Kang, S. Jiang, H. Berger, K. Watanabe, T. Taniguchi, L. Forró, J. Shan, and K. F. Mak, Giant anisotropic magnetoresistance in Ising superconductor-magnetic insulator tunnel junctions (2021), arXiv:2101.01327

[24] H. Idzuchi, F. Pientka, K.-F. Huang, K. Harada, O. Gül, Y. J. Shin, L. T. Nguyen, N. H. Jo, D. Shindo, R. J. Cava, P. C. Canfield, and P. Kim, Van der Waals heterostructure magnetic Josephson junction (2020), arXiv:2012.14969.

[25] L. Ai, E. Zhang, C. Huang, X. Xie, Y. Yang, Z. Jia, Y. Zhang, S. Liu, Z. Li, P. Leng, X. Sun, X. Kou, Z. Han, and F. Xiu, Van der Waals ferromagnetic Josephson junctions (2021), arXiv:2101.04323.

[26] M. Tinkham, Introduction to superconductivity (Dover Publications, Mineola, 2004).

[27] L. N. Bulaevskii, A. A. Guseinov, and A. I. Rusinov, Superconductivity in crystals without symmetry centers, Sov. Phys. JETP 44, 1243 (1976), [Zh. Eksp. Teor. Fiz. 71, 2356 (1976)].

[28] L. P. Gor'kov and E. I. Rashba, Superconducting 2D system with lifted spin degeneracy: Mixed singlet-triplet state, Phys. Rev. Lett. 87, 037004 (2001)

[29] P. A. Frigeri, D. F. Agterberg, A. Koga, and M. Sigrist, Superconductivity without inversion symmetry: $\mathrm{MnSi}$ versus $\mathrm{CePt}_{3} \mathrm{Si}$, Phys. Rev. Lett. 92, 097001 (2004)

[30] B. S. Chandrasekhar, A note on the maximum critical field of high-field superconductors, Applied Physics Letters 1, 7 (1962)

[31] A. M. Clogston, Upper limit for the critical field in hard superconductors, Phys. Rev. Lett. 9, 266 (1962)

[32] S. Ilić, J. S. Meyer, and M. Houzet, Enhancement of the upper critical field in disordered transition metal dichalcogenide monolayers, Phys. Rev. Lett. 119, 117001 (2017)

[33] D. Möckli and M. Khodas, Robust parity-mixed superconductivity in disordered monolayer transition metal dichalcogenides, Phys. Rev. B 98, 144518 (2018)

[34] D. Möckli and M. Khodas, Magnetic-field induced $s+$ if pairing in Ising superconductors, Phys. Rev. B 99, 180505(R) (2019)

[35] D. Möckli and M. Khodas, Ising superconductors: Interplay of magnetic field, triplet channels, and disorder, Phys. Rev. B 101, $014510(2020)$

[36] D. Möckli, M. Haim, and M. Khodas, Magnetic impurities in thin films and 2D Ising superconductors, Journal of Applied Physics 128, 053903 (2020)

[37] M. Haim, D. Möckli, and M. Khodas, Signatures of triplet correlations in density of states of ising superconductors, Phys. Rev. B 102, 214513 (2020)

[38] H. Liu, H. Liu, D. Zhang, and X. C. Xie, Microscopic theory of in-plane critical field in two-dimensional Ising superconducting systems, Phys. Rev. B 102, 174510 (2020)

[39] M. A. Rahimi, A. G. Moghaddam, C. Dykstra, M. Governale, and U. Zülicke, Unconventional superconductivity from magnetism in transition-metal dichalcogenides, Phys. Rev. B 95, 104515 (2017)

[40] D. Wickramaratne, S. Khmelevskyi, D. F. Agterberg, and I. I. Mazin, Ising superconductivity and magnetism in $\mathrm{NbSe}_{2}$, Phys. Rev. X 10, 041003 (2020)

[41] W.-Y. He, B. T. Zhou, J. J. He, N. F. Q. Yuan, T. Zhang, and K. T. Law, Magnetic field driven nodal topological superconductivity in monolayer transition metal dichalcogenides, Communications Physics 1, 40 (2018). 
[42] Y. Xie, B. T. Zhou, T. K. Ng, and K. T. Law, Strongly enlarged topological regime and enhanced superconducting gap in nanowires coupled to Ising superconductors, Phys. Rev. Research 2, 013026 (2020)

[43] O. Lesser, G. Shavit, and Y. Oreg, Topological superconductivity in carbon nanotubes with a small magnetic flux, Phys. Rev. Research 2, 023254 (2020)

[44] P. Lv, Y.-F. Zhou, N.-X. Yang, and Q.-F. Sun, Magnetoanisotropic spin-triplet Andreev reflection in ferromagnetIsing superconductor junctions, Phys. Rev. B 97, 144501 (2018)

[45] Q. Cheng and Q.-F. Sun, Switch effect and 0- $\pi$ transition in Ising superconductor Josephson junctions, Phys. Rev. B 99, 184507 (2019)

[46] See Supplemental Material for derivations and additional details.

[47] V. L. Berezinskii, New model of the anisotropic phase of superfluid $\mathrm{He}^{3}$, JETP Lett. 20, 287 (1974), [Pis'ma Zh. Eksp. Teor. Fiz. 20, 628-631 (1974)].

[48] Y. Tanaka, M. Sato, and N. Nagaosa, Symmetry and topology in superconductors-odd-frequency pairing and edge states-, Journal of the Physical Society of Japan 81, 011013 (2012).

[49] Y. Fukaya, K. Yada, A. Hattori, and Y. Tanaka, Pairing mechanism of unconventional superconductivity in doped kane-mele model, Journal of the Physical Society of Japan 85, 104704 (2016)

[50] J. Linder and A. V. Balatsky, Odd-frequency superconductivity, Rev. Mod. Phys. 91, 045005 (2019)

[51] G. Eilenberger, Transformation of Gorkov's equation for type II superconductors into transport-like equations, Zeitschrift für Physik A Hadrons and nuclei 214, 195 (1968)

[52] A. I. Larkin and Y. N. Ovchinnikov, Quasiclassical method in the theory of superconductivity, JETP 28, 1200 (1969), [Zh. Eksp. Teor. Fiz. 55, 2262-2272 (1968)].

[53] W. Belzig, F. K. Wilhelm, C. Bruder, G. Schön, and A. D. Zaikin, Quasiclassical Green's function approach to mesoscopic superconductivity, Superlattices Microstruct. 25, 1251 (1999).

[54] N. Kopnin, Theory of Nonequilibrium Superconductivity (Oxford University Press, 2001).

[55] M. Eschrig, Spin-polarized supercurrents for spintronics: a review of current progress, Reports on Progress in Physics 78, 104501 (2015)

[56] P. Anderson, Theory of dirty superconductors, Journal of Physics and Chemistry of Solids 11, 26 (1959)

[57] R. C. Dynes, J. P. Garno, G. B. Hertel, and T. P. Orlando, Tunneling study of superconductivity near the metal-insulator transition, Phys. Rev. Lett. 53, 2437 (1984)

[58] L. Komendová, A. V. Balatsky, and A. M. Black-Schaffer, Experimentally observable signatures of odd-frequency pairing in multiband superconductors, Phys. Rev. B 92, 094517 (2015).

[59] P. Fulde and R. A. Ferrell, Superconductivity in a strong spinexchange field, Phys. Rev. 135, A550 (1964).

[60] A. I. Larkin and Y. N. Ovchinnikov, Nonuniform state of superconductors, JETP 20, 762 (1965), [Zh. Eksp. Teor. Fiz. 47, 1136-1146 (1964)].

[61] S. Diesch, P. Machon, M. Wolz, C. Sürgers, D. Beckmann, W. Belzig, and E. Scheer, Creation of equal-spin triplet superconductivity at the $\mathrm{Al} / \mathrm{EuS}$ interface, Nat. Comm. 9, 5248 (2018). 


\section{Supplemental Material for "Magnetic field-induced 'mirage' gap in an Ising superconductor"}

\section{PAIRING-CORRELATION FUNCTION}

We consider the effective mean-field Hamiltonian near the $K$ or $K^{\prime}$ valley with

$$
H_{\mathrm{BdG}}=\left[\begin{array}{cc}
H_{0}(\boldsymbol{k}) & \Delta i \sigma_{y} \\
-\Delta i \sigma_{y} & -H_{0}^{*}(-\boldsymbol{k})
\end{array}\right]
$$

in the Nambu basis $\left(c_{\boldsymbol{k}, \uparrow}, c_{\boldsymbol{k}, \downarrow}, c_{-\boldsymbol{k}, \uparrow}^{\dagger}, c_{-\boldsymbol{k}, \downarrow}^{\dagger}\right)$. The Hamiltonian $H_{0}$ is

$$
H_{0}(\boldsymbol{k}=\boldsymbol{p}+s \boldsymbol{K})=\xi_{\boldsymbol{p}} \sigma_{0}+s \beta_{\mathrm{so}} \sigma_{z}-\boldsymbol{B} \cdot \boldsymbol{\sigma}
$$

where $s \boldsymbol{K}$ is the momentum of the $K(s=+)$ or $K^{\prime}(s=-)$ valley and $\boldsymbol{p}$ is the momentum deviation from $\boldsymbol{K}$ or $-\boldsymbol{K}$. Furthermore, $\xi_{\boldsymbol{p}}=|\boldsymbol{p}|^{2} /(2 m)-\mu$ is the dispersion measured form the chemical potential $\mu$. The Pauli matrices $\sigma_{x}, \sigma_{y}$, and $\sigma_{z}$ act on the spin space and $\sigma_{0}$ is the corresponding unit matrix. In this work, we also define the Pauli matrices $\tau_{1}, \tau_{2}$ and $\tau_{3}$ acting on the Nambu space with the corresponding unit matrix $\tau_{0}$. The Ising spin-orbit coupling (ISOC) strength is denoted as $\beta_{\text {so }}$. The Zeeman term is $-\boldsymbol{B} \cdot \boldsymbol{\sigma}$ where the magnetic field is in the $x-z$ plane and parametrized as

$$
\boldsymbol{B}=\left(B_{x}, 0, B_{z}\right)=B(\sin \theta, 0, \cos \theta) .
$$

The magnetic field $\boldsymbol{B}$ absorbs the prefactor $g_{L} \mu_{B}$ with the Landé g-factor $g_{L}$ and the Bohr magneton $\mu_{B}$.

The pairing-correlation function $F(\boldsymbol{k}, \varepsilon)$ with the form

$$
F(\boldsymbol{k}, \varepsilon)=\Delta\left[F_{0}(\boldsymbol{k}, \varepsilon) \sigma_{0}+\boldsymbol{F}(\boldsymbol{k}, \varepsilon) \cdot \boldsymbol{\sigma}\right] i \sigma_{y},
$$

can be obtained from the Gor'kov equation [54]

$$
\left[\begin{array}{cc}
\varepsilon-H_{0}(\boldsymbol{k}) & -\Delta i \sigma_{y} \\
\Delta i \sigma_{y} & \varepsilon+H_{0}^{*}(-\boldsymbol{k})
\end{array}\right]\left[\begin{array}{c}
F(\boldsymbol{k}, \varepsilon) \\
\bar{G}(\boldsymbol{k}, \varepsilon)
\end{array}\right]=\left[\begin{array}{l}
0 \\
1
\end{array}\right]
$$

One can obtain

$$
\begin{aligned}
& F_{0}(\boldsymbol{k}, \varepsilon)=\left(\varepsilon^{2}-\xi_{\boldsymbol{p}}^{2}-\Delta^{2}-\beta_{\mathrm{SO}}^{2}+B^{2}\right) / M(\boldsymbol{k}, \varepsilon), \\
& F_{x}(\boldsymbol{k}, \varepsilon)=-2 \varepsilon B_{x} / M(\boldsymbol{k}, \varepsilon), \\
& F_{y}(\boldsymbol{k}, \varepsilon)=-2 i s \beta_{\mathrm{so}} B_{x} / M(\boldsymbol{k}, \varepsilon), \\
& F_{z}(\boldsymbol{k}, \varepsilon)=-2\left(\varepsilon B_{z}-s \beta_{\mathrm{so}} \xi_{\boldsymbol{p}}\right) / M(\boldsymbol{k}, \varepsilon),
\end{aligned}
$$

with

$$
\begin{aligned}
M(\boldsymbol{k}, \varepsilon) & =\left(\varepsilon^{2}-\xi_{\boldsymbol{p}}^{2}-\Delta^{2}-\beta_{\mathrm{so}}^{2}+B^{2}\right)^{2} \\
& -4\left(\varepsilon B_{z}-s \beta_{\mathrm{so}} \xi_{\boldsymbol{p}}\right)^{2}-4 B_{x}^{2}\left(\varepsilon^{2}-\beta_{\mathrm{so}}^{2}\right)
\end{aligned}
$$

\section{TRANSFORMATION OF SPIN-QUANTIZATION AXIS}

We consider a general spin-quantization orientation parametrized by the polar angle $(\theta)$ and azimuthal angle $(\varphi)$ with respect to the $z$-axis. The transformation from the spinquantization axis characterized by orientation $(\theta, \varphi)$ to that by $z$-axis is given by

$$
\left[\begin{array}{l}
|\uparrow\rangle_{\theta, \varphi} \\
|\downarrow\rangle_{\theta, \varphi}
\end{array}\right]=\left[\begin{array}{cc}
\cos (\theta / 2) & \sin (\theta / 2) \\
-\sin (\theta / 2) & \cos (\theta / 2)
\end{array}\right]\left[\begin{array}{l}
e^{-i \varphi / 2}|\uparrow\rangle \\
e^{+i \varphi / 2}|\downarrow\rangle
\end{array}\right],
$$

where $|\uparrow\rangle$ and $|\downarrow\rangle$ are spin vectors in the $z$-axis. This transformation rule applies to the Cooper pairs as well, for example,

$$
\begin{aligned}
(|\uparrow \downarrow\rangle-|\downarrow \uparrow\rangle)_{\theta, \varphi}= & |\uparrow \downarrow\rangle-|\downarrow \uparrow\rangle, \\
(|\uparrow \downarrow\rangle+|\downarrow \uparrow\rangle)_{\theta, \varphi}= & -\sin \theta\left[e^{-i \varphi}|\uparrow \uparrow\rangle-e^{i \varphi}|\downarrow \downarrow\rangle\right] \\
& +\cos \theta[|\uparrow \downarrow\rangle+|\downarrow \uparrow\rangle] .
\end{aligned}
$$

Specifically, the transformations of pairing forms from the $x$ axis with $\theta=\pi / 2$ and $\varphi=0$ to the $z$-axis are

$$
\begin{aligned}
& (|\uparrow \downarrow\rangle-|\downarrow \uparrow\rangle)_{x}=|\uparrow \downarrow\rangle-|\downarrow \uparrow\rangle, \\
& (|\uparrow \downarrow\rangle+|\downarrow \uparrow\rangle)_{x}=|\downarrow \downarrow\rangle-|\uparrow \uparrow\rangle, \\
& (|\uparrow \uparrow\rangle+|\downarrow \downarrow\rangle)_{x}=|\uparrow \uparrow\rangle+|\downarrow \downarrow\rangle, \\
& (|\uparrow \uparrow\rangle-|\downarrow \downarrow\rangle)_{x}=|\uparrow \downarrow\rangle+|\downarrow \uparrow\rangle .
\end{aligned}
$$

\section{QUASICLASSICAL GREEN'S FUNCTION}

The quasiclassical formalism concentrates on the phenomena close to the Fermi surface [51-54], it can be applied to the situation where both the superconducting gap and the ISOC are much smaller than the Fermi energy. The structure of the quasiclassical Green's function is [54, 55]

$$
\hat{g}(\hat{\boldsymbol{k}}, \varepsilon)=\left[\begin{array}{cc}
g_{0} \sigma_{0}+\boldsymbol{g} \cdot \boldsymbol{\sigma} & \left(f_{0} \sigma_{0}+\boldsymbol{f} \cdot \boldsymbol{\sigma}\right) i \sigma_{y} \\
\left(\bar{f}_{0} \sigma_{0}+\overline{\boldsymbol{f}} \cdot \boldsymbol{\sigma}^{*}\right) i \sigma_{y} & \bar{g}_{0} \sigma_{0}+\overline{\boldsymbol{g}} \cdot \boldsymbol{\sigma}^{*}
\end{array}\right],
$$

where $\hat{\boldsymbol{k}}$ denotes the direction of momentum $\boldsymbol{k}$ and the bar operation is defined as $\bar{q}(\hat{\boldsymbol{k}}, \varepsilon)=q\left(-\hat{\boldsymbol{k}},-\varepsilon^{*}\right)^{*}$ with $q \in$ $\left\{g_{0}, f_{0}, \boldsymbol{g}, \boldsymbol{f}, \Delta, \boldsymbol{\Delta}, \boldsymbol{\nu}\right\}$. We introduce the notation $\boldsymbol{g}_{ \pm}=$ $(\boldsymbol{g} \pm \overline{\boldsymbol{g}}) / 2$. The anomalous Green's functions $f_{0}$ and $\boldsymbol{f}$ characterize the singlet and triplet pairings, respectively. The requirement that $\operatorname{Tr}(\hat{g})=0$ leads to $\bar{g}_{0}=-g_{0}$. The normalization condition $\hat{g} \hat{g}=\sigma_{0} \tau_{0}$ gives

$$
\begin{aligned}
& g_{0}^{2}+\boldsymbol{g}_{+}^{2}+\boldsymbol{g}_{-}^{2}-f_{0} \bar{f}_{0}+\boldsymbol{f} \cdot \overline{\boldsymbol{f}}=1, \\
& 2 g_{0} \boldsymbol{g}_{+}=\bar{f}_{0} \boldsymbol{f}-f_{0} \overline{\boldsymbol{f}}, \\
& 2 g_{0} \boldsymbol{g}_{-}=i \overline{\boldsymbol{f}} \times \boldsymbol{f} .
\end{aligned}
$$

with $\boldsymbol{g}_{ \pm}^{2}=\boldsymbol{g}_{ \pm, x}^{2}+\boldsymbol{g}_{ \pm, y}^{2}+\boldsymbol{g}_{ \pm, z}^{2}$.

The quasiclassical Green's function $\hat{g}(\hat{\boldsymbol{k}}, \varepsilon)$ obeys the Eilenberger equation [51, 54],

$$
\left[\varepsilon \sigma_{0} \tau_{3}-\hat{\Delta}-\hat{\nu}-\hat{\Sigma}(\varepsilon), \hat{g}\right]+i \hbar \boldsymbol{v}_{F} \cdot \nabla_{\boldsymbol{R}} \hat{g}=0
$$


Here, the order parameter term $\hat{\Delta}$ is explicitly written as

$$
\hat{\Delta}=\left[\begin{array}{ll}
\left(\Delta \sigma_{0}+\boldsymbol{\Delta} \cdot \boldsymbol{\sigma}\right) i \sigma_{y} \\
\left(\bar{\Delta} \sigma_{0}+\overline{\boldsymbol{\Delta}} \cdot \boldsymbol{\sigma}^{*}\right) i \sigma_{y}
\end{array} .\right.
$$

The ISOC and Zeeman fields are included in $\hat{\nu}$ with

$$
\hat{\nu}=\left[\begin{array}{cc}
\boldsymbol{\nu} \cdot \boldsymbol{\sigma} & \\
& \overline{\boldsymbol{\nu}} \cdot \boldsymbol{\sigma}^{*}
\end{array}\right]=s \beta_{\mathrm{so}} \sigma_{z} \tau_{3}-\left[\begin{array}{cc}
\boldsymbol{B} \cdot \boldsymbol{\sigma} & \\
& \boldsymbol{B} \cdot \boldsymbol{\sigma}^{*}
\end{array}\right] .
$$

By introducing the notation $\boldsymbol{\nu}_{ \pm}=(\boldsymbol{\nu} \pm \overline{\boldsymbol{\nu}}) / 2$, we have

$$
\hat{\nu}=\left[\begin{array}{ll}
\left(\boldsymbol{\nu}_{+}+\boldsymbol{\nu}_{-}\right) \cdot \boldsymbol{\sigma} & \\
& \left(\boldsymbol{\nu}_{+}-\boldsymbol{\nu}_{-}\right) \cdot \boldsymbol{\sigma}^{*}
\end{array}\right],
$$

with

$$
\begin{array}{ll}
\nu_{+, x}=-B_{x}, & \nu_{+, z}=-B_{z}, \\
\nu_{-, x}=0, & \nu_{-, z}=s \beta_{\mathrm{so}} .
\end{array}
$$

The nonmagnetic impurities are considered within the selfconsistent Born approximation with

$$
\hat{\Sigma}(\varepsilon)=-i \Gamma\langle\hat{g}(\hat{\boldsymbol{k}}, \varepsilon)\rangle,
$$

where $\Gamma$ is the intervalley impurity scattering strength and $\langle\cdots\rangle$ denotes averaging over the whole Fermi momentum direction.

\section{CLEAN LIMIT}

For a homogeneous system $\left(\nabla_{\boldsymbol{R}} \hat{g}=0\right)$ in the clean limit $(\Gamma=0)$, the Eilenberger equation, Eq. (31), is reduced to

$$
\left[\varepsilon \hat{\tau}_{3}-\hat{\Delta}-\hat{\nu}, \hat{g}\right]=0
$$

The off-diagonal terms in the Nambu space produce

$$
\begin{aligned}
& \varepsilon f_{0}-\boldsymbol{\nu}_{+} \cdot \boldsymbol{f}+\Delta g_{0}+\boldsymbol{\Delta} \cdot \boldsymbol{g}_{+}=0 \\
& \varepsilon \boldsymbol{f}-f_{0} \boldsymbol{\nu}_{+}-i \boldsymbol{\nu}_{-} \times \boldsymbol{f}+g_{0} \boldsymbol{\Delta}+\Delta \boldsymbol{g}_{+}+i \boldsymbol{g}_{-} \times \boldsymbol{\Delta}=0 \\
& -\varepsilon \bar{f}_{0}-\boldsymbol{\nu}_{+} \cdot \overline{\boldsymbol{f}}-\bar{\Delta} g_{0}+\overline{\boldsymbol{\Delta}} \cdot \boldsymbol{g}_{+}=0, \\
& -\varepsilon \overline{\boldsymbol{f}}-\bar{f}_{0} \boldsymbol{\nu}_{+}-i \boldsymbol{\nu}_{-} \times \overline{\boldsymbol{f}}-g_{0} \overline{\boldsymbol{\Delta}}+\bar{\Delta} \boldsymbol{g}_{+}+i \boldsymbol{g}_{-} \times \overline{\boldsymbol{\Delta}}=0 .
\end{aligned}
$$

In combination with Eqs. (28)-(30), one can obtain all the components of the quasiclassical Green's function $\hat{g}$. Considering a Hamiltonian with only an $s$-wave singlet pairing $(\Delta=0)$, Eqs. 39-42 are reduced to

$$
\begin{aligned}
& \varepsilon f_{0}-\boldsymbol{\nu}_{+} \cdot \boldsymbol{f}+\Delta e^{i \phi} g_{0}=0 \\
& \varepsilon \boldsymbol{f}-f_{0} \boldsymbol{\nu}_{+}-i \boldsymbol{\nu}_{-} \times \boldsymbol{f}+\Delta e^{i \phi} \boldsymbol{g}_{+}=0 \\
& \varepsilon \bar{f}_{0}+\boldsymbol{\nu}_{+} \cdot \overline{\boldsymbol{f}}+\Delta e^{-i \phi} g_{0}=0 \\
& \varepsilon \overline{\boldsymbol{f}}+\bar{f}_{0} \boldsymbol{\nu}_{+}+i \boldsymbol{\nu}_{-} \times \overline{\boldsymbol{f}}-\Delta e^{-i \phi} \boldsymbol{g}_{+}=0
\end{aligned}
$$

where $\phi$ is the superconducting phase. Equations (43) and 44) can be written explicitly as

$$
\left[\begin{array}{cccc}
\varepsilon & B_{x} & 0 & B_{z} \\
B_{x} & \varepsilon & i s \beta_{\mathrm{so}} & 0 \\
0 & -i s \beta_{\mathrm{so}} & \varepsilon & 0 \\
B_{z} & 0 & 0 & \varepsilon
\end{array}\right]\left[\begin{array}{c}
f_{0} \\
f_{x} \\
f_{y} \\
f_{z}
\end{array}\right]+\Delta e^{i \phi}\left[\begin{array}{c}
g_{0} \\
g_{+, x} \\
g_{+, y} \\
g_{+, z}
\end{array}\right]=0 .
$$

We now try to obtain some relations between $\left(f_{0}, \boldsymbol{f}\right)$ and $\left(\bar{f}_{0}, \overline{\boldsymbol{f}}\right)$. By combining Eqs. 43, and 45, we have

$$
e^{-i \phi}\left(\varepsilon f_{0}-\boldsymbol{\nu}_{+} \cdot \boldsymbol{f}\right)=e^{i \phi}\left(\varepsilon \bar{f}_{0}+\boldsymbol{\nu}_{+} \cdot \overline{\boldsymbol{f}}\right) .
$$

By combining Eqs. 44, and 46, we have

$$
e^{-i \phi}\left(\varepsilon \boldsymbol{f}-f_{0} \boldsymbol{\nu}_{+}-i \boldsymbol{\nu}_{-} \times \boldsymbol{f}\right)=-e^{i \phi}\left(\varepsilon \overline{\boldsymbol{f}}-\bar{f}_{0} \boldsymbol{\nu}_{+}+i \boldsymbol{\nu}_{-} \times \overline{\boldsymbol{f}}\right) .
$$

The above two equations can be explicitly written as

$$
\begin{aligned}
& \varepsilon\left(f_{0}-\bar{f}_{0}\right)+B_{x}\left(f_{x}+\bar{f}_{x}\right)+B_{z}\left(f_{z}+\bar{f}_{z}\right)=0, \\
& \varepsilon\left(f_{x}+\bar{f}_{x}\right)+B_{x}\left(f_{0}-\bar{f}_{0}\right)+i s \beta_{\mathrm{so}}\left(f_{y}-\bar{f}_{y}\right)=0, \\
& \varepsilon\left(f_{y}+\bar{f}_{y}\right)-i s \beta_{\mathrm{so}}\left(f_{x}-\bar{f}_{x}\right)=0, \\
& \varepsilon\left(f_{z}+\bar{f}_{z}\right)+B_{z}\left(f_{0}-\bar{f}_{0}\right)=0,
\end{aligned}
$$

where the phase factors $e^{-i \phi}$ and $e^{i \phi}$ are absorbed into $\left(f_{0}, \boldsymbol{f}\right)$ and $\left(\bar{f}_{0}, \overline{\boldsymbol{f}}\right)$, respectively. From Eqs. 50 - 53, we have

$$
f_{0}=\bar{f}_{0}, \quad f_{x}=-\bar{f}_{x}, \quad f_{y}=\bar{f}_{y}, \quad f_{z}=-\bar{f}_{z}
$$

and

$$
\varepsilon f_{y}=i s \beta_{\text {so }} f_{x} .
$$

Equation (54) results in $g_{ \pm, y}=0$ from Eqs. 29) and 30. Then Eqs. 28-30 can be written explicitly as

$$
\begin{gathered}
g_{0}^{2}+g_{+, x}^{2}+g_{+, z}^{2}+g_{-, x}^{2}+g_{-, z}^{2}-f_{0}^{2}-f_{x}^{2}+f_{y}^{2}-f_{z}^{2}=1, \\
g_{0} g_{+, x}=f_{0} f_{x}, \quad g_{0} g_{+, z}=f_{0} f_{z}, \\
g_{0} g_{-, x}=+i f_{y} f_{z}, \quad g_{0} g_{-, z}=-i f_{x} f_{y} .
\end{gathered}
$$

In the following, we obtain the corresponding components of the Green's function $\hat{g}$ from Eq. (47) and Eqs. 54-58 for three different cases.

For the case of a magnetic field with an arbitrary direction, we can express

$$
f_{x}=a \varepsilon B_{x}, \quad f_{y}=a i s \beta_{\mathrm{so}} B_{x}, \quad f_{z}=b \varepsilon B_{z},
$$

where $a$ and $b$ are to be fixed by the normalization condition, Eq. (56). From Eqs. (47) and (57), we can obtain

$$
(a-b) f_{0}=-a b \beta_{\mathrm{so}}^{2},
$$

where $a$ and $b$ satisfy

$$
\begin{aligned}
& \quad a^{2} b \beta_{\mathrm{so}}^{4}-a(a-b)\left(b \varepsilon^{2}+a B_{x}^{2}+b B_{z}^{2}-b \Delta^{2}\right) \beta_{\mathrm{so}}^{2} \\
& +(a-b)^{2} \varepsilon^{2}\left(a B_{x}^{2}+b B_{z}^{2}\right)=0 .
\end{aligned}
$$


Then $g_{0}$ can be obtained from the first relation in Eq. 477, which is

$$
\varepsilon f_{0}+B_{x} f_{x}+B_{z} f_{z}+\Delta g_{0}=0 .
$$

The terms $g_{+, x}$ and $g_{+, z}$ can be obtained from Eq. [57], and $g_{-, x}$ and $g_{-, z}$ from Eq. 58.

For the case with an out-of-plane magnetic field $B_{z}$, both the $x$ - and $y$-components are decoupled so that $f_{x}=f_{y}=$ $g_{+, x}=g_{+, y}=0$, which leads to $\boldsymbol{g}_{-}=0$ from Eq. (58). From Eqs. (47) and (57) and the normalization condition, Eq. (56), we can directly get the Green's functions. By defining $f_{\uparrow(\downarrow)}=$ $f_{0} \pm f_{z}$ and $g_{\uparrow(\downarrow)}=g_{0} \pm g_{+, z}$, so that $f_{0(z)}=\left(f_{\uparrow} \pm f_{\downarrow}\right) / 2$ and $g_{0(+, z)}=\left(g_{\uparrow} \pm g_{\downarrow}\right) / 2$, we have

$$
g_{\uparrow(\downarrow)}=\frac{\varepsilon \pm B_{z}}{\sqrt{\left(\varepsilon \pm B_{z}\right)^{2}-\Delta^{2}}}, \quad f_{\uparrow(\downarrow)}=\frac{-\Delta}{\sqrt{\left(\varepsilon \pm B_{z}\right)^{2}-\Delta^{2}}} .
$$

For the case of an in-plane magnetic field $B_{x}$, one can see from Eq. (47) that both $f_{z}$ and $g_{+, z}$ are decoupled from the other components so that $f_{z}=g_{+, z}=0$. We have

$$
f_{x}=a \varepsilon B_{x}, \quad f_{y}=a i s \beta_{\mathrm{so}} B_{x} .
$$

From Eqs. (47) and (57), the expressions of $g_{0}$ and $f_{0}$ are obtained as

$$
\begin{aligned}
& g_{0}=a \varepsilon c /(2 \Delta), \\
& f_{0}=-a\left(B_{x}^{2}+c / 2\right),
\end{aligned}
$$

where

$$
c=\varepsilon^{2}-\beta_{\mathrm{so}}^{2}-B_{x}^{2}-\Delta^{2}-u
$$

with

$$
u= \pm \sqrt{\left(\varepsilon^{2}-\beta_{\mathrm{so}}^{2}-B_{x}^{2}-\Delta^{2}\right)^{2}-4 B_{x}^{2} \Delta^{2}} .
$$

The terms $g_{+, x}$ and $g_{-, z}$ can be obtained from Eqs. (57) and (58), respectively. The coefficient $a$ can be fixed by the normalization condition

$$
g_{0}^{2}+g_{+, x}^{2}+g_{-, z}^{2}-f_{0}^{2}-f_{x}^{2}+f_{y}^{2}=1,
$$

so that

$a^{2}=\frac{4 c^{2} \Delta^{2}}{\left[4 \Delta^{2} B_{x}^{2}\left(B_{x}^{2}+\beta_{\mathrm{so}}^{2}+c\right)+c^{2}\left(\Delta^{2}-\varepsilon^{2}\right)\right]\left(4 \Delta^{2} B_{x}^{2}-c^{2}\right)}$,

which can be further simplified as

$$
a^{2}=\frac{c \Delta^{2}}{u^{2}\left[c\left(\varepsilon^{2}-\Delta^{2}\right)-2 \Delta^{2} B_{x}^{2}\right]} .
$$

Since $a$ is even in energy and does not depend on the valley index $s, f_{x}$ is even in valley index and odd in energy, while $f_{y}$ is odd in valley index and even in energy.

In Fig. 33, we show various components of the Green's function at different in-plane magnetic fields in the clean limit. In
Fig. 4(a), we show the effects of the magnetic field direction on the DOS with the magnitudes of the magnetic field fixed. The out-of-plane component causes the spin splitting near the superconducting gap and the mirage gaps are not influenced. The effects of the magnetic field magnitude with a fixed direction are shown in Fig. 4 (b) where we can see that the mirage gap depends on the magnetic field magnitude.

\section{NONMAGNETIC IMPURITY EFFECT}

The Eilenberger equation for a homogeneous system with impurities reads

$$
\left[\varepsilon \hat{\tau}_{3}-\hat{\Delta}-\hat{\nu}-\hat{\Sigma}(\varepsilon), \hat{g}\right]=0,
$$

with $\hat{\Sigma}(\varepsilon)=-i \Gamma\langle\hat{g}(\hat{\boldsymbol{k}}, \varepsilon)\rangle$ where $\Gamma$ is the nonmagnetic intervalley impurity scattering rate. Considering an in-plane magnetic field, we have $f_{z}=0$. Due to the fact that $f_{y}$ and $g_{-, z}$ are odd with respect to the valley index $s$, we have $\left\langle f_{y}\right\rangle=$ $\left\langle g_{-, z}\right\rangle=0$. Since the other components of the quasi-classical Green's function are independent of the momentum, the average notation $\langle\cdots\rangle$ can be omitted in $\hat{\Sigma}(\varepsilon)=-i \Gamma\langle\hat{g}(\hat{\boldsymbol{k}}, \varepsilon)\rangle$. The Eilenberger equation can be recast into

$$
\left[\tilde{\varepsilon} \sigma_{0} \tau_{3}-\hat{\tilde{\Delta}}-\hat{\tilde{\nu}}, \hat{g}\right]=0
$$

with $\tilde{\varepsilon}=\varepsilon+i \Gamma g_{0}$. The effective superconducting gap is

$$
\hat{\tilde{\Delta}}=\left[\begin{array}{ll} 
& \left(\tilde{\Delta} \sigma_{0}+\tilde{\boldsymbol{\Delta}} \cdot \boldsymbol{\sigma}\right) i \sigma_{y} \\
\left(\overline{\tilde{\Delta}} \sigma_{0}+\overline{\tilde{\Delta}} \cdot \boldsymbol{\sigma}^{*}\right) i \sigma_{y} &
\end{array}\right],
$$

with $\tilde{\Delta}=\Delta e^{i \phi}-i \Gamma f_{0}, \tilde{\Delta}_{x}=-i \Gamma f_{x}, \overline{\tilde{\Delta}}=\Delta e^{-i \phi}-i \Gamma \bar{f}_{0}$, $\overline{\tilde{\Delta}}_{x}=-i \Gamma \bar{f}_{x}$, and $\tilde{\Delta}_{y}=\tilde{\Delta}_{z}=\overline{\tilde{\Delta}}_{y}=\overline{\tilde{\Delta}}_{z}=0$. We also have

$$
\hat{\tilde{\nu}}=\left[\begin{array}{ll}
\left(\tilde{\boldsymbol{\nu}}_{+}+\tilde{\boldsymbol{\nu}}_{-}\right) \cdot \boldsymbol{\sigma} & \\
& \left(\tilde{\boldsymbol{\nu}}_{+}-\tilde{\boldsymbol{\nu}}_{-}\right) \cdot \boldsymbol{\sigma}^{*}
\end{array}\right],
$$

with

$$
\begin{array}{ll}
\tilde{\nu}_{+, x}=-\tilde{B}_{x}, & \tilde{\nu}_{+, z}=0, \\
\tilde{\nu}_{-, x}=0, & \tilde{\nu}_{-, z}=s \beta_{\mathrm{so}},
\end{array}
$$

where $\tilde{B}_{x}=B_{x}+i \Gamma g_{+, x}$.

Equations 397-42 become

$$
\begin{aligned}
& \tilde{\varepsilon} f_{0}-\tilde{\boldsymbol{\nu}}_{+} \cdot \boldsymbol{f}+\tilde{\Delta} g_{0}+\tilde{\boldsymbol{\Delta}} \cdot \boldsymbol{g}_{+}=0, \\
& \tilde{\varepsilon} \boldsymbol{f}-f_{0} \tilde{\boldsymbol{\nu}}_{+}-i \tilde{\boldsymbol{\nu}}_{-} \times \boldsymbol{f}+g_{0} \tilde{\boldsymbol{\Delta}}+\tilde{\Delta} \boldsymbol{g}_{+}=0, \\
& \tilde{\varepsilon} \bar{f}_{0}+\tilde{\boldsymbol{\nu}}_{+} \cdot \overline{\boldsymbol{f}}+\tilde{\tilde{\Delta}} g_{0}-\overline{\tilde{\Delta}} \cdot \boldsymbol{g}_{+}=0, \\
& \tilde{\varepsilon} \overline{\boldsymbol{f}}+\bar{f}_{0} \tilde{\boldsymbol{\nu}}_{+}+i \tilde{\boldsymbol{\nu}}_{-} \times \overline{\boldsymbol{f}}+g_{0} \overline{\tilde{\Delta}}-\overline{\tilde{\Delta}} \boldsymbol{g}_{+}=0,
\end{aligned}
$$

which can be explicitly written as

$$
\left[\begin{array}{ccc}
\tilde{\varepsilon} & \tilde{B}_{x} & 0 \\
\tilde{B}_{x} & \tilde{\varepsilon} & i s \beta_{\mathrm{so}} \\
0 & -i s \beta_{\mathrm{so}} & \tilde{\varepsilon}
\end{array}\right]\left[\begin{array}{c}
f_{0} \\
f_{x} \\
f_{y}
\end{array}\right]+\left[\begin{array}{ccc}
\tilde{\Delta} & \tilde{\Delta}_{x} & 0 \\
\tilde{\Delta}_{x} & \tilde{\Delta} & 0 \\
0 & 0 & \tilde{\Delta}
\end{array}\right]\left[\begin{array}{c}
g_{0} \\
g_{+, x} \\
0
\end{array}\right]=0
$$



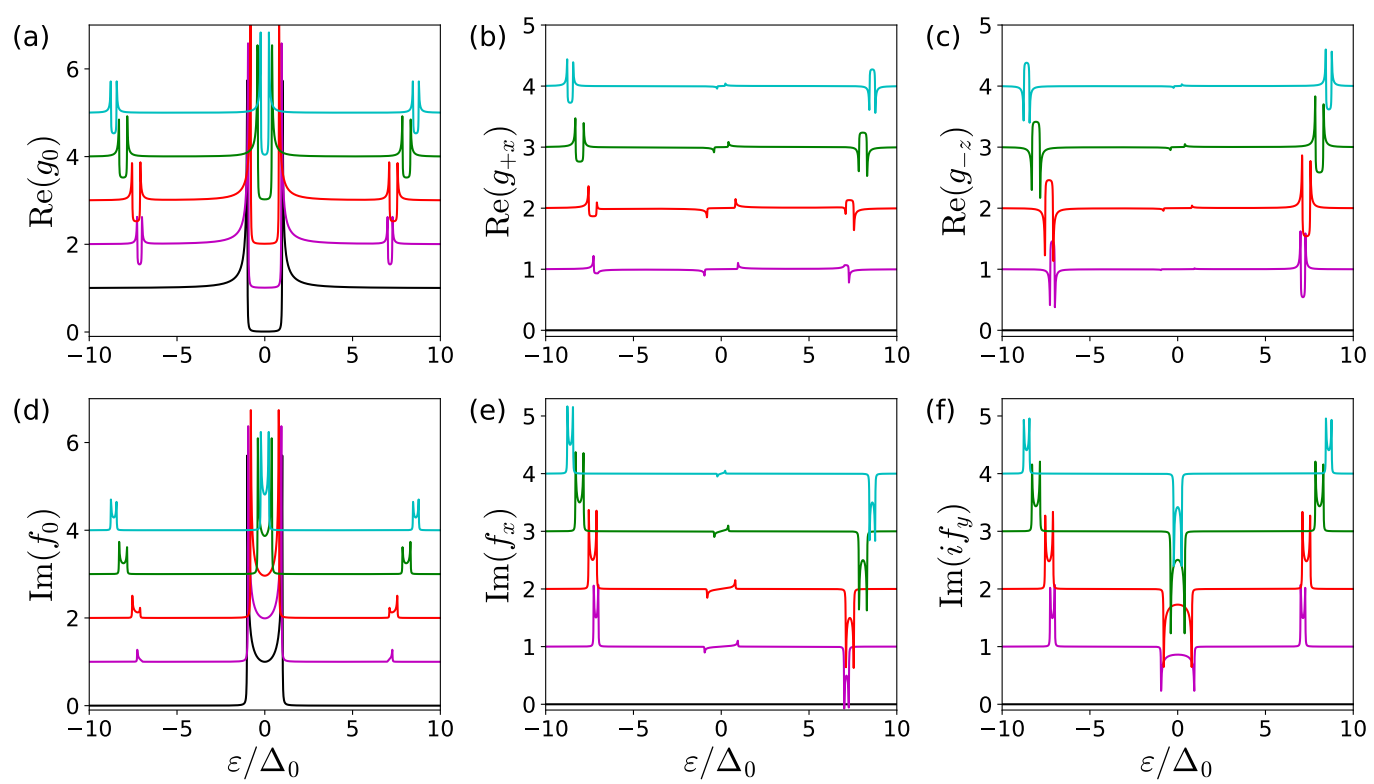

FIG. 3. Various components of the Green's function at different in-plane magnetic fields (from bottom to top: $B_{x}=0, \Delta_{0}, 2 \Delta_{0}, 4 \Delta_{0}, 5 \Delta_{0}$ ) in the clean limit. All curves for finite $B_{x}$ have been offset vertically for better visibility. Here, $\beta_{\text {so }}=7 \Delta_{0}$ and $T=0.1 T_{c 0}$.
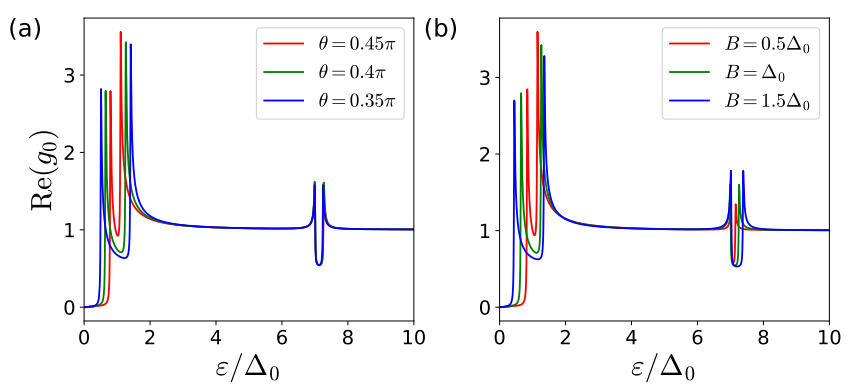

FIG. 4. Density of states $\operatorname{Re}\left(g_{0}\right)$ (a) at different $\theta$ with $B=\Delta_{0}$ and (b) at different $B$ with $\theta=0.4 \pi$ in the clean limit. Here, $\beta_{\text {so }}=7 \Delta_{0}$ and $T=0.1 T_{c}$.

and

$$
\left[\begin{array}{ccc}
\tilde{\varepsilon} & -\tilde{B}_{x} & 0 \\
\tilde{B}_{x} & -\tilde{\varepsilon} & i s \beta_{\mathrm{so}} \\
0 & -i s \beta_{\mathrm{so}} & -\tilde{\varepsilon}
\end{array}\right]\left[\begin{array}{c}
\bar{f}_{0} \\
\bar{f}_{x} \\
\bar{f}_{y}
\end{array}\right]+\left[\begin{array}{ccc}
\overline{\tilde{\Delta}} & -\overline{\tilde{\Delta}}_{x} & 0 \\
-\overline{\tilde{\Delta}}_{x} & \overline{\tilde{\Delta}} & 0 \\
0 & 0 & \tilde{\tilde{\Delta}}
\end{array}\right]\left[\begin{array}{c}
g_{0} \\
g_{+, x} \\
0
\end{array}\right]=0 .
$$

From these equations, we still have the relations

$$
f_{0}=\bar{f}_{0}, \quad f_{x}=-\bar{f}_{x}, \quad f_{y}=\bar{f}_{y},
$$

and

$$
\tilde{\varepsilon} f_{y}=i s \beta_{\mathrm{so}} f_{x} .
$$

Similarly to Eq. 63, we can assume $f_{x}$ and $f_{y}$ as

$$
f_{x}=a \tilde{\varepsilon} B_{x}, \quad f_{y}=a i s \beta_{\mathrm{so}} B_{x},
$$

where $a$ is fixed by the normalization condition,

$$
g_{0}^{2}+g_{+, x}^{2}+g_{-, z}^{2}-f_{0}^{2}-f_{x}^{2}+f_{y}^{2}=1 .
$$

From Eqs. (77) and 29], which is $g_{0} g_{+, x}=f_{0} f_{x}$, we have

$$
g_{0}=a \varepsilon \frac{\varepsilon^{2}-\beta_{\mathrm{so}}^{2}-B_{x}^{2}-\Delta^{2}-2 i \Gamma a B_{x}^{2} \Delta-u}{2 \Delta\left(1-\Gamma^{2} a^{2} B_{x}^{2}\right)-2 i \Gamma a\left(\varepsilon^{2}-B_{x}^{2}-\Delta^{2}\right)} \equiv a \varepsilon \frac{c}{d},
$$

where

$$
u= \pm \sqrt{\left(\varepsilon^{2}-\beta_{\mathrm{so}}^{2}-B_{x}^{2}-\Delta^{2}\right)^{2}-4 B_{x}^{2}\left(\Delta^{2}-i \Gamma a \Delta \beta_{\mathrm{so}}^{2}\right)} .
$$

Using the normalization condition, we have

$$
\begin{aligned}
& a^{2}\left\{\left[B_{x}^{2}(d+i \Gamma c a)+c \Delta\right]^{2}-c^{2} \varepsilon^{2}+B_{x}^{2} \beta_{\mathrm{so}}^{2} d^{2}\right\} \times \\
& {\left[B_{x}^{2}(d+i \Gamma c a)^{2}-c^{2}\right]-c^{2} d^{2}=0}
\end{aligned}
$$

Our task now is to get $a$ from Eq. 85].

Since $a \sim \Delta /\left(\varepsilon \beta_{\mathrm{so}}^{2}\right)$ and we are mainly interested in the DOS for $|\varepsilon| \gtrsim \Delta$, the quantity $\Gamma a B_{x}$ can be considered to be small under the condition $\Gamma B_{x} \ll \beta_{\mathrm{so}}^{2}$. Considering a small intervalley scattering strength $\Gamma$ with $\Gamma B_{x} \ll \beta_{\mathrm{so}}^{2}$, one has

$$
g_{0} \approx a \varepsilon \frac{\left(\varepsilon^{2}-\beta_{\mathrm{so}}^{2}-B_{x}^{2}-\Delta^{2}\right)-u}{2 \Delta-2 i \Gamma a\left(\varepsilon^{2}-B_{x}^{2}-\Delta^{2}\right)}
$$

with $u \approx \pm \sqrt{\left(\varepsilon^{2}-\beta_{\text {so }}^{2}-B_{x}^{2}-\Delta^{2}\right)^{2}-4 B_{x}^{2} \Delta^{2}}$. Equation (85) reduces to a polynomial equation of degree six with respect to $a$. To obtain the coefficients of the polynomial using Mathematica, we write $d+i \Gamma c a=2 \Delta-i \Gamma x a$ with $x=\varepsilon^{2}-B_{x}^{2}-\Delta^{2}+\beta_{\mathrm{so}}^{2}+u$ and $d=2 \Delta-i \Gamma y a$ with 


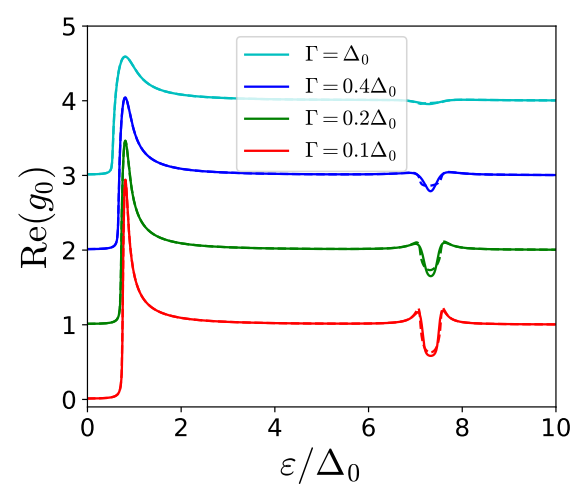

FIG. 5. Comparison of the density of states between the exact (solid lines) and approximate results (dashed lines) for different impurity scattering strengths. Here, $\beta_{\mathrm{so}}=7 \Delta_{0}, B_{x}=2 \Delta_{0}$ and $T=0.1 T_{c 0}$.

$y=2\left(\varepsilon^{2}-B_{x}^{2}-\Delta^{2}\right)$, so that

$$
\begin{aligned}
& \Gamma^{4} B_{x}^{4} x^{2}\left(B_{x}^{2} x^{2}+\beta_{\mathrm{so}}^{2} y^{2}\right) a^{6} \\
+ & 2 i B_{x}^{4} \Gamma^{3} \Delta x\left[\left(4 B_{x}^{2}+c\right) x^{2}+2 y(x+y) \beta_{\mathrm{so}}^{2}\right] a^{5} \\
+ & B_{x}^{2} \Gamma^{2}\left[c^{2} x^{2}\left(\varepsilon^{2}+B_{x}^{2}-\Delta^{2}\right)+\beta_{\mathrm{so}}^{2} c^{2} y^{2}\right. \\
& \left.-12 B_{x}^{2} \Delta^{2} x^{2}\left(2 B_{x}^{2}+c\right)-4 B_{x}^{2} \Delta^{2} \beta_{\mathrm{so}}^{2}\left(x^{2}+4 x y+y^{2}\right)\right] a^{4} \\
- & 2 i \Gamma B_{x}^{2} \Delta\left[4 B_{x}^{2} \Delta^{2} x\left(4 B_{x}^{2}+3 c\right)+8 B_{x}^{2} \beta_{\mathrm{so}}^{2} \Delta^{2}(x+y)\right. \\
& \left.-c^{2} x\left(2 \varepsilon^{2}+2 B_{x}^{2}-2 \Delta^{2}+c\right)-2 c^{2} \beta_{\mathrm{so}}^{2} y\right] a^{3} \\
+ & \left\{\Gamma^{2} c^{2} y^{2}+\left[4 \Delta^{2} B_{x}^{2}\left(B_{x}^{2}+\beta_{\mathrm{so}}^{2}+c\right)+c^{2}\left(\Delta^{2}-\varepsilon^{2}\right)\right]\right. \\
& \left.\left(4 \Delta^{2} B_{x}^{2}-c^{2}\right)\right\} a^{2}+4 i \Gamma c^{2} y \Delta a-4 c^{2} \Delta^{2}=0 .
\end{aligned}
$$

An exact solution for the coefficient $a$ can be numerically obtained using NLsolve.jl package in Julia where the initial value can be assigned using the approximated solution in the above equation. The terms $f_{0}, g_{+, x}$ and $g_{-, z}$ can be obtained from

$$
\begin{aligned}
& \varepsilon f_{0}+a \varepsilon B_{x}^{2}+\left(i \Gamma a B_{x}^{2}+\Delta\right) g_{0}=0, \\
& g_{0} g_{+, x}=f_{0} f_{x}, \\
& g_{0} g_{-, z}=-i f_{x} f_{y},
\end{aligned}
$$

respectively. The comparison between the approximate solution using Eq. 87) and the exact result for the density of states is shown in Fig. 5

\section{GAP EQUATION}

The superconducting gap is determined from the selfconsistent gap equation

$$
\frac{\Delta}{\lambda}=-2 i \pi T \sum_{n=0}^{N_{c}} f_{0}\left(i \omega_{n}\right)
$$

where $\lambda$ is the dimensionless coupling constant and $\omega_{n}=$ $(2 n+1) \pi k_{B} T$ the Matsubara frequency. The summation cutoff $N_{c}$ is determined by the cutoff frequency $\Omega_{c}$ with $N_{c}=\left\lfloor\hbar \Omega_{c} /\left(2 \pi k_{B} T\right)\right\rfloor$. The term of $f_{0}\left(i \omega_{n}\right)$ is obtained
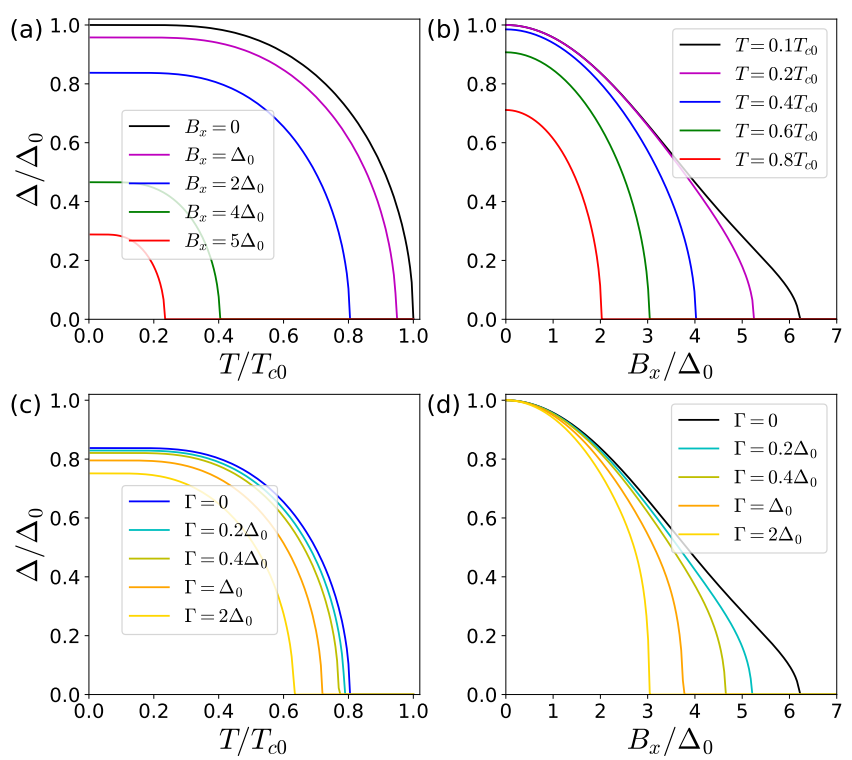

FIG. 6. (a) Superconducting gap $\Delta$ versus temperature $T$ at different magnetic fields $B_{x}$. (b) Gap $\Delta$ versus $B_{x}$ at different temperatures. Panels (a) and (b) are in the clean limit. (c) Gap $\Delta$ versus $T$ at different intervalley scattering strengths $\Gamma$ with $B_{x}=2 \Delta_{0}$. (d) Gap $\Delta$ versus $B_{x}$ at different $\Gamma$ with $T=0.1 T_{c}$. Here, $\beta_{\text {so }}=7 \Delta_{0}$.

from $f(\varepsilon)$ by replacing $\varepsilon$ with $i \omega_{n}$. In the clean limit without a magnetic field, we have $\hbar \Omega_{c}=\Delta_{0} \sinh (1 / \lambda)$ [26], where $\Delta_{0}$ is the zero-temperature gap in the absence of a magnetic field. The behaviors of the superconducting gap $\Delta$ are shown in Fig. 6 .

With $\Delta \rightarrow 0$ in Eq. 83 , one has $u=-\left(\varepsilon^{2}-\beta_{\text {so }}^{2}-B_{x}^{2}\right)$ so that Eq. (83) can be written as

$$
g_{0}=a \varepsilon \frac{\varepsilon^{2}-\beta_{\mathrm{so}}^{2}-B_{x}^{2}}{\Delta\left(1-\Gamma^{2} a^{2} B_{x}^{2}\right)-i \Gamma a\left(\varepsilon^{2}-B_{x}^{2}-\Delta^{2}\right)} .
$$

Since $g_{0} \approx 1$ under $\Delta \rightarrow 0$, one has

$$
a \approx \frac{\Delta}{(\varepsilon+i \Gamma)\left(\varepsilon^{2}-B_{x}^{2}\right)-\varepsilon \beta_{\mathrm{so}}^{2}},
$$

so that

$$
f_{0}(\varepsilon)=\Delta \frac{-\varepsilon(\varepsilon+i \Gamma)+\beta_{\mathrm{so}}^{2}}{(\varepsilon+i \Gamma)\left(\varepsilon^{2}-B_{x}^{2}-\beta_{\mathrm{so}}^{2}\right)+i \Gamma \beta_{\mathrm{so}}^{2}} .
$$

Using the relation [35]

$$
-\frac{1}{\lambda}=\ln \left(\frac{T}{T_{c 0}}\right)+2 \pi T \sum_{n=0}^{\infty} \frac{1}{\omega_{n}},
$$

where $T_{c 0}$ is the zero-field critical temperature, the in-plane critical magnetic field $B_{c}$ can be found through the pairbreaking equation

$$
\ln \left(\frac{T}{T_{c 0}}\right)=2 \pi T \sum_{n=0}^{\infty}\left[\frac{\omega_{n} \tilde{\omega}_{n}+\beta_{\mathrm{so}}^{2}}{\tilde{\omega}_{n}\left(\omega_{n}^{2}+B_{c}^{2}+\beta_{\mathrm{so}}^{2}\right)-\Gamma \beta_{\mathrm{so}}^{2}}-\frac{1}{\omega_{n}}\right],
$$




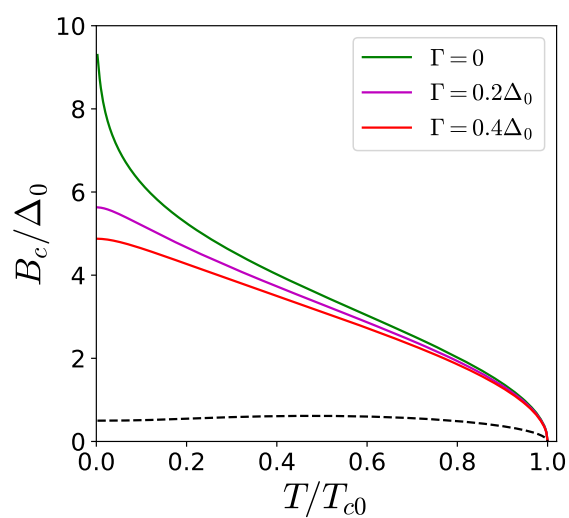

FIG. 7. Critical in-plane magnetic field $B_{c}$ versus temperature $T$ at different intervalley scattering strengths $\Gamma$ with $\beta_{\text {so }}=7 \Delta_{0}$. The out-of-plane critical magnetic field is shown as the dashed line. with $\tilde{\omega}_{n}=\omega_{n}+\Gamma$. This expression agrees with Refs. [32, 35]. The in-plane critical magnetic fields at different intervalley scattering strengths are shown in Fig. 7 